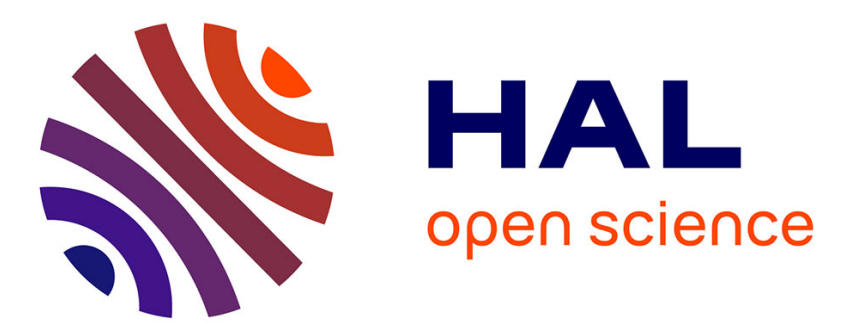

\title{
Circularly polarized few-optical-cycle solitons in Kerr media: A complex modified Korteweg-de Vries model
}

Hervé Leblond, Houria Triki, François Sanchez, Dumitru Mihalache

\section{To cite this version:}

Hervé Leblond, Houria Triki, François Sanchez, Dumitru Mihalache. Circularly polarized few-opticalcycle solitons in Kerr media: A complex modified Korteweg-de Vries model. Optics Communications, 2012, 285 (3), pp.356 - 363. 10.1016/j.optcom.2011.09.051 . hal-03187686

\section{HAL Id: hal-03187686 \\ https://univ-angers.hal.science/hal-03187686}

Submitted on 1 Apr 2021

HAL is a multi-disciplinary open access archive for the deposit and dissemination of scientific research documents, whether they are published or not. The documents may come from teaching and research institutions in France or abroad, or from public or private research centers.
L'archive ouverte pluridisciplinaire HAL, est destinée au dépôt et à la diffusion de documents scientifiques de niveau recherche, publiés ou non, émanant des établissements d'enseignement et de recherche français ou étrangers, des laboratoires publics ou privés. 


\title{
Circularly polarized few-optical-cycle solitons in Kerr media: A complex modified Korteweg-de Vries model
}

\author{
H. Leblond ${ }^{\text {a,* }}$, H. Triki ${ }^{\text {b }}$, F. Sanchez ${ }^{\text {a }}$, D. Mihalache ${ }^{\text {a,c,d }}$ \\ a Laboratoire de Photonique d'Angers, EA 4464, Université d'Angers, 2 Bd. Lavoisier, 49045 Angers Cedex 01, France \\ ${ }^{\mathrm{b}}$ Radiation Physics Laboratory, Department of Physics, Faculty of Sciences, Badji Mokhtar University, P. O. Box 12, 23000 Annaba, Algeria \\ ${ }^{c}$ Horia Hulubei National Institute for Physics and Nuclear Engineering, P.O.B. MG-6, 077125 Magurele, Romania \\ d Academy of Romanian Scientists, 54 Splaiul Independentei, Bucharest 050094, Romania
}

\section{A R T I C L E I N F O}

\section{Article history:}

Received 17 August 2011

Received in revised form 19 September 2011

Accepted 20 September 2011

Available online 6 October 2011

Keywords:

Few-cycle pulses

Few-cycle solitons

Circular polarization

Modified Korteweg-de Vries equation

mKdV equation

\begin{abstract}
A B S T R A C T
We consider the propagation of few-cycle pulses (FCPs) in cubic nonlinear media exhibiting a "crystal-like" structure, beyond the slowly varying envelope approximation, taking into account the wave polarization. By using the reductive perturbation method we derive from the Maxwell-Bloch-Heisenberg equations, in the long-wave-approximation regime, a non-integrable complex modified Korteweg-de Vries equation describing the propagation of circularly polarized (CP) FCPs. By direct numerical simulations of the governing nonlinear partial differential equation we get robust CP FCPs and we show that the unstable ones decays into linearly polarized half-cycle pulses, whose polarization direction slowly rotates around the propagation axis.
\end{abstract}

(c) 2011 Elsevier B.V. All rights reserved.

\section{Introduction}

Interest in intense ultrashort light pulses containing a few optical cycles has grown steadily in recent years since their first experimental realization in 1999 [1-4]. This mature research area has considerable potential for ultrafast optics applications in metrology of ultrafast phenomena, in systems performing laser ablation (micromachining, etching, microsurgery), etc. It still presents many exciting open problems from both a fundamental and applied point of view. Notably, the ultrashort pulses possess extensive applications to the field of light-matter interactions, high-order harmonic generation, extreme [5] and single-cycle [6] nonlinear optics, and attosecond physics [7]; for a review of earlier works in this area see Ref. [8].

Recent progress in the study of the wave dynamics of few-cycle pulses (FCPs) in nonlinear optical media has paved the way for the development of new theoretical approaches to model their propagation in physical systems. Three classes of main dynamical models for FCPs have been put forward: (i) the quantum approach [9-12], (ii) the refinements within the framework of the slowly varying envelope approximation (SVEA) of the nonlinear Schrödinger-type envelope equations [13-16], and the non-SVEA models [17-25]. Other recent works on FCPs deals with few-cycle light bullets created by

\footnotetext{
* Corresponding author. Tel.: + 332417354 31; fax: +332 41735216 . E-mail address: herve.leblond@univ-angers.fr (H. Leblond).
}

femtosecond filaments [26], the study of ultrashort spatiotemporal optical solitons in quadratic nonlinear media [27], the ultrashort spatiotemporal optical pulse propagation in cubic (Kerr-like) media without the use of the SVEA [28], single-cycle gap solitons generated in resonant two-level dense media with a subwavelength structure [29], observation of few-cycle propagating surface plasmon polariton wavepackets [30], the possibility of generating few-cycle dissipative optical solitons [31,32], and the existence of guided optical solitons of femtosecond duration and nanoscopic mode area, that is, femtosecond nanometer-sized optical solitons [33].

We also mention recent studies of ultrafast pulse propagation in mode-locked laser cavities in the few femtosecond pulse regime and the derivation of a master mode-locking equation for ultrashort pulses [34]. Another relevant recent work presents a class of few-cycle elliptically polarized solitary waves in isotropic Kerr media, proposes a method of producing multisolitons with different polarization states, and study their binary-collision dynamics [35].

The propagation of FCPs in Kerr media can be described beyond the SVEA by using the modified Korteweg-de Vries (mKdV) [19], sine-Gordon (sG) [20,21], or mKdV-sG equations [22,23]. The mKdV and $\mathrm{sG}$ equations are completely integrable by means of the inverse scattering transform (IST) method [36,37], whereas the mKdV-sG equation is completely integrable only if some condition between its coefficients is satisfied [38].

The aim of this work is to give a comprehensive study of the propagation of circularly polarized (CP) few-optical-cycle pulses in cubic (Kerr-like) nonlinear media beyond SVEA and when the frequency 
of the transition is far above the characteristic wave frequency (the so-called long-wave approximation regime). The present work completes our previous brief study [39] and it gives all necessary physical and mathematical details of the governing model, together with an alternative derivation of it, in the relevant physical situation of a "crystal-like" system. Notice that CP FCPs have been recently considered in Ref. [40] in the so-called short-wave approximation regime, i.e., when the frequency of the transition is far below the characteristic wave frequency. We therefore focus on the vectorial feature of the electric field by taking into account the wave polarization effects. We show, by using the reductive perturbation method (multiscale analysis), that the evolution of the two wave amplitudes describing the vectorial character of ultrashort optical pulse propagation in a system of two-level atoms in cubic nonlinear media is well described by two coupled complex mKdV equations at the third-order limit of perturbation approach. This result is of great interest since it shows that vector solitons in cubic (Kerr-like) media beyond the SVEA behave as those in a nonlinear medium that is modeled by the KdV-type equation when the order $\varepsilon^{3}$ of perturbation limit is applied to the Maxwell-Bloch-Heisenberg equations.

The paper is organized as follows. In the next section we derive the dynamical equations governing the propagation of vector femtosecond optical solitons by developing multiple scaling approach to the Maxwell-Bloch-Heisenberg equations up to the third order in the small parameter $\varepsilon$. The basic model used in the present work is a system of two-level atoms in the relevant case of a "crystal-like" system, where the excited level is twice degenerated, with the induced dipole oriented either in the $x$ or in the $y$ direction. In Section 3 we analyze the main features of the obtained complex mKdV equation. Then in Section 4 we obtain some analytical approximate solutions for CP few-optical-cycle solitons which are valid for long pulses. We also get by direct numerical simulations, robust CP FCPs and we describe the transition of unstable $\mathrm{CP}$ few-optical-cycle solitons to stable linearly polarized (LP) single-humped (half-cycle) pulses. We also show that the wave profile of this half-cycle pulse accurately coincides with that of the fundamental soliton of the real mKdV equation and that this single pulse performs a slow rotation around the propagation axis. Finally, in Section 5 we summarize the results of our analysis and we indicate some possible extensions of this work.

\section{Derivation of a model}

\subsection{Basic equations: a crystal}

A system of two coupled mKdV equations was derived in [39] from a model of a glass, or an amorphous medium. This model involved two-level atoms with Hamiltonian

$H_{0}=\hbar\left(\begin{array}{cc}\omega_{a} & 0 \\ 0 & \omega_{b}\end{array}\right)$

and some induced dipolar electric momentum $\vec{\mu}$, oriented randomly in the transverse plane $(x, y)$, as

$\vec{\mu}=\mu\left(\cos \theta \overrightarrow{e_{x}}+\sin \theta \overrightarrow{e_{y}}\right)$

where $\overrightarrow{e_{x}}$ and $\overrightarrow{e_{y}}$ are the unitary vectors along the $x$ axis and $y$ axis, respectively, and

$\mu=\left(\begin{array}{cc}0 & \mu \\ \mu^{*} & 0\end{array}\right)$

The polarization density was given by

$\vec{P}=N\langle\operatorname{Tr}(\rho \vec{\mu})\rangle$ where $N$ is the number of atoms per unit volume, $\rho$ is the density matrix, and $\langle\cdot\rangle$ denotes the averaging over all directions in the $x-y$ plane.

We will show below that the same governing equations can be derived from another model, which would rather correspond to a crystalline structure.

This alternative approach involves a two-level medium, in which the excited level is twice degenerated, with the induced dipole oriented either in the $x$ or in the $y$ direction. Precisely, the Hamiltonian is

$H_{0}=\hbar\left(\begin{array}{ccc}\omega_{a} & 0 & 0 \\ 0 & \omega_{b} & 0 \\ 0 & 0 & \omega_{b}\end{array}\right)$

still with $\Omega=\omega_{b}-\omega_{a}>0$. The dipolar momentum becomes

$\vec{\mu}=\mu_{x} \overrightarrow{e_{x}}+\mu_{y} \overrightarrow{e_{y}}$

where

$\mu_{x}=\left(\begin{array}{ccc}0 & \mu & 0 \\ \mu^{*} & 0 & 0 \\ 0 & 0 & 0\end{array}\right)$,

$\mu_{y}=\left(\begin{array}{ccc}0 & 0 & \mu \\ 0 & 0 & 0 \\ \mu^{*} & 0 & 0\end{array}\right)$.

The evolution of the electric field $\vec{E}$ is governed by the Maxwell equations which, in the absence of magnetic effects, and assuming a plane wave propagating along the $z$ axis, reduce to

$\partial_{z}^{2} \vec{E}=\frac{1}{c^{2}} \partial_{t}^{2}(\vec{E}+4 \pi \vec{P})$

where $\vec{P}$ is the polarization density. It is given by

$\vec{P}=N \operatorname{Tr}(\rho \vec{\mu})$

where $N$ is the number of atoms per unit volume and $\rho$ is the density matrix.

The evolution of the density-matrix is governed by the Heisenberg equation

$i \hbar \partial_{t} \rho=[H, \rho]$,

where

$H=H_{0}-\vec{\mu} \cdot \vec{E}$

describes the coupling between the atoms and the electric field. The relaxation effects can be neglected here as in the scalar approximation, see [20]. Notice that the physical values of the relaxation times are indeed in the picosecond range, or even slower (nanoseconds), thus very large with regard to the pulse duration, which allows us to neglect them.

\subsection{Evolution equations in the long-wave approximation}

Since transparency is required for soliton propagation, the typical frequency $\omega_{\mathrm{w}}$ of the wave must be far away from the resonance frequency $\Omega$. Far away means either much lower or much higher. In the present paper, we restrict to the first situation, $\omega_{\mathrm{w}} \ll \Omega$. If $\omega_{\mathrm{w}}$ is in the visible range, it assumes that the transition frequency is in the ultraviolet. The typical length of the wave, say $t_{\mathrm{w}}=1 / \omega_{\mathrm{w}}$, is very large with respect to the characteristic time $t_{\mathrm{r}}=1 / \Omega$ associated to the transition. It is thus a long wave approximation, as defined in the framework of the reductive perturbation method [41,42]. 
It may seem strange that we use a long-wave approximation to describe ultrashort pulses. Recall however that the word 'long' is here relative to some reference value of the wavelength. The latter is the characteristic wavelength $\lambda_{r}=c t_{r}=2 \pi c / \Omega$ of the transition, which belongs to the ultraviolet spectral range, i.e. much smaller than the characteristic wavelength $\lambda_{w}$ of the ultrashort pulse belonging to the visible spectral range, which can therefore be considered as being comparatively 'long' $\left(\lambda_{r} \ll \lambda_{w}\right)$. The main application of the long-wave approximation is the hydrodynamic KdV soliton, which is a solitary wave in the sense that it contains only a single oscillation. More generally, such a formalism is suited to the investigation of the exact wave profile, when the wave packet contains few oscillations and the use of wave envelope is not adequate. This is the case we study here.

We introduce a small parameter $\varepsilon$, which can be here $\varepsilon=1$ / $\left(\Omega t_{\mathrm{w}}\right) \ll 1$, and the slow variables

$\tau=\varepsilon\left(t-\frac{z}{V}\right), \quad \zeta=\varepsilon^{3} z$

The retarded time variable $\tau$ describes the pulse shape, propagating at speed $V$ in a first approximation. Its order of magnitude $\varepsilon$ gives an account of the long-wave approximation, so that the corresponding values of retarded time have the same order of magnitude as $t_{\mathrm{r}} / \varepsilon=$ $t_{\mathrm{w}} \gg t_{\mathrm{r}}$ if $\tau$ is of the order of unity. The propagation distance is assumed to be very long with regard to the pulse length $c t_{\mathrm{w}}$; therefore it will have the same order of magnitude as $c t_{\mathrm{r}} / \varepsilon^{n}$, where $n \geqslant 2$. The value of $n$ is determined by the distance at which dispersion effects occur. According to the general theory of the derivation of KdV-type equations [42], it is $n=3$. The $\zeta$ variable of order $\varepsilon^{3}$ describes thus long-distance propagation.

The electric field $\vec{E}$, the polarization density $\vec{P}$, and the density matrix $\rho$ are expanded in power series of $\varepsilon$ as

$\vec{E}=\sum_{n \geq 1} \varepsilon^{n} \vec{E}_{n}=\sum_{n \geq 1} \varepsilon^{n}\left(u_{n}, v_{n}, 0\right)$,

$\vec{P}=\sum_{n \geq 1} \varepsilon^{n}\left(P_{n}, Q_{n}, 0\right)$,

$\rho=\sum_{n \geq 0} \varepsilon^{n} \rho_{n}$

in which the triplets of coordinates are given in the $(x, y, z)$ frame, and the profiles $u_{1}, v_{1}$, etc., are functions of the slow variables $\tau$ and $\zeta$. The components of $\rho^{n}$ are denoted by $\rho_{i j}^{n}$.

At lowest order $\varepsilon^{1}$, the Heisenberg Eq. (11) yields

$\rho_{12}^{1}=\frac{\mu}{\hbar \Omega} u_{1}, \quad \rho_{13}^{1}=\frac{\mu}{\hbar \Omega} v_{1}$,

and consequently

$P_{1}=\frac{2 N|\mu|^{2}}{\hbar \Omega} u_{1}$

$Q_{1}=\frac{2 N|\mu|^{2}}{\hbar \Omega} v_{1}$

which are the same expressions as in the glass model [39], except that $N$ is replaced with $2 N$.

At order $\varepsilon^{3}$ in the Maxwell Eq. (9), we get an expression of the refractive index with the same slight change:

$n=\left(1+\frac{8 \pi N|\mu|^{2}}{\hbar \Omega}\right)^{\frac{1}{2}}$
At order $\varepsilon^{2}$ in the Heisenberg Eq. (11), we first notice that $\partial_{\tau} \rho_{23}^{1}=0$ and consequently $\rho_{23}^{1}=0$. In the same way, $\rho_{11}^{1}=\rho_{22}^{1}=\rho_{33}^{1}=0$. Then we get

$\rho_{12}^{2}=\frac{\mu}{\hbar \Omega} u_{2}-\frac{i \mu}{\hbar \Omega^{2}} \partial_{\tau} u_{1}$

$\rho_{13}^{2}=\frac{\mu}{\hbar \Omega} v_{2}-\frac{i \mu}{\hbar \Omega^{2}} \partial_{\tau} v_{1}$

which are the same expressions as in the case of the "glass" model [39], with the orientation angle of the dipolar momentum $\theta=0$ for $\rho_{12}^{2}$ and $\theta=\pi / 2$ for $\rho_{13}^{2}$. Consequently, we get

$P_{2}=\frac{2 N|\mu|^{2}}{\hbar \Omega} u_{2}$

$Q_{2}=\frac{2 N|\mu|^{2}}{\hbar \Omega} v_{2}$,

and the Maxwell equation at order $\varepsilon^{4}$ is automatically satisfied.

At order $\varepsilon^{3}$ in the Heisenberg Eq. (11), the populations are computed as

$\rho_{11}^{2}=\frac{-|\mu|^{2}}{\hbar^{2} \Omega^{2}}\left(u_{1}^{2}+v_{1}^{2}\right)$

$\rho_{22}^{2}=\frac{|\mu|^{2}}{\hbar^{2} \Omega^{2}} u_{1}^{2}$

$\rho_{33}^{2}=\frac{|\mu|^{2}}{\hbar^{2} \Omega^{2}} v_{1}^{2}$.

Notice that a nonzero coherence term between the two excited states appears, it is

$\rho_{23}^{2}=\frac{|\mu|^{2}}{\hbar^{2} \Omega^{2}} u_{1} v_{1}$

Consequently, the coherence between the fundamental state and the state excited in the $x$ direction at next order is

$$
\begin{aligned}
\rho_{12}^{3}= & \frac{\mu}{\hbar \Omega} u_{3}-\frac{i \mu}{\hbar \Omega^{2}} \partial_{\tau} u_{2} \\
& -\frac{\mu}{\hbar \Omega^{3}} \partial_{\tau}^{2} u_{1}-\frac{2 \mu|\mu|^{2}}{\hbar^{3} \Omega^{3}}\left(u_{1}^{2}+v_{1}^{2}\right) u_{1} .
\end{aligned}
$$

The analogous expression, permuting $u_{1}$ and $v_{1}$, is obtained for the component $\rho_{13}^{3}$.

The expressions for the polarization density components $P_{3}$ and $Q_{3}$ are obtained, as

$P_{3}=\frac{2 N|\mu|^{2}}{\hbar \Omega} u_{3}-\frac{2 N|\mu|^{2}}{\hbar \Omega^{3}} \partial_{\tau}^{2} u_{1}-\frac{4 N|\mu|^{4}}{\hbar^{3} \Omega^{3}}\left(u_{1}^{2}+v_{1}^{2}\right) u_{1}$,

and analogously for $Q_{3}$. Apart from the change from $N$ to $2 N$ already noticed, the only discrepancy with respect to the corresponding equations in the glass model [39] is a coefficient value 4 instead of 3 in the nonlinear term in Eq. (30).

The Maxwell Eq. (9) at order $\varepsilon^{5}$ yields the following pair of coupled equations:

$$
\begin{aligned}
& \partial_{\zeta} u_{1}=A \partial_{\tau}^{3} u_{1}+B \partial_{\tau}\left[\left(u_{1}^{2}+v_{1}^{2}\right) u_{1}\right] \\
& \partial_{\zeta} v_{1}=A \partial_{\tau}^{3} v_{1}+B \partial_{\tau}\left[\left(u_{1}^{2}+v_{1}^{2}\right) v_{1}\right]
\end{aligned}
$$


in which we have set

$A=\frac{4 \pi N|\mu|^{2}}{n c \hbar \Omega^{3}}$

$B=\frac{8 \pi N|\mu|^{4}}{n c \hbar^{3} \Omega^{3}}$

Notice that the structure of the set of Eqs. (31), (32) is the same as in the "glass" model [39], with very slightly modified coefficients. The expressions of the two dispersion coefficients coincide (the ratio between the corresponding dispersion coefficients is therefore 1) if we consider that only in the case of the "glass-like" model, one half of the dipoles only are contributing, while all of them are involved in the "crystal-like" model. The same feature is observed in the case of the refractive index. Regarding the value (34) of the coefficient $B$, the ratio between the corresponding nonlinear coefficients is a bit smaller, $3 / 8$, which is nothing else but the average value $\left\langle\cos ^{4} \theta\right\rangle$ of $\cos ^{4} \theta$, which is involved in the averaging of the nonlinear polarization density over all orientations $\theta$ of $\vec{\mu}$ in the glass model [39]. In fact, the coefficients $A$ and $B$ have here exactly the same expressions as in the scalar model [20].

Eqs. $(31,32)$ can be written in the normalized form as

$U_{Z}=U_{T T T}+\left[\left(U^{2}+V^{2}\right) U\right]_{T}$

$V_{Z}=V_{T T T}+\left[\left(U^{2}+V^{2}\right) V\right]_{T}$,

where the subscripts $Z$ and $T$ denote the derivatives, and the functions and variables are defined as

$U=\frac{u_{1}}{\mathcal{E}}, \quad V=\frac{v_{1}}{\mathcal{E}}, \quad Z=\frac{z}{\mathcal{L}}, \quad T=\frac{t-z / V}{t_{\mathrm{w}}}$,

with

$\mathcal{L}=\frac{n \hbar c \Omega^{3} t_{\mathrm{w}}^{3}}{2 \pi N|\mu|^{2}}$

$\mathcal{E}=\sqrt{\frac{2}{3} \frac{\hbar}{|\mu| t_{\mathrm{w}}}}$

Eqs. $(35,36)$ are a set of coupled mKdV equations describing the propagation of optical FCPs in a medium presenting cubic nonlinearity and dispersion [43]. They can be also seen as describing the interaction of two linearly polarized FCPs, $U$ and $V$.

\section{The complex mKdV equation}

Assuming that $U$ and $V$ vanish at infinity, the mKdV system (35)-(36) has four conserved quantities [44]:

$I_{1}=\int_{-\infty}^{+\infty} U d T, \quad I_{2}=\int_{-\infty}^{+\infty} V d T$,

the momentum of the system

$I_{3}=\int_{-\infty}^{+\infty}\left(U^{2}+V^{2}\right) d T$,

and its Hamiltonian

$I_{4}=\frac{1}{2} \int_{-\infty}^{+\infty}\left[\left(U^{2}+V^{2}\right)^{2}-2\left(U_{T}^{2}+V_{T}^{2}\right)\right] d T$,

which remain constant with $\mathrm{Z}$.
Setting

$f=U+i V$

Eqs. (35) and (36) reduce to

$f_{Z}=f_{T T T}+\left(|f|^{2} f\right)_{T}$

which is known as the complex modified Korteweg-de Vries (cmKdV) equation. Confusion must be avoided between Eq. (44) and the other cmKdV equation

$f_{Z}=f_{T T T}+\left(|f|^{2}\right) f_{T}$.

Indeed, Eq. (45) is completely integrable [45] while Eq. (44) is not. Eqs. (44) and (45) are sometimes referred to as cmKdV I and cmKdV II equations, respectively. The integrable Eq. (45) has been extensively studied (see e.g. [45-48]), while less studies have been devoted to the non-integrable Eq. (44)[49,50]. In Ref. [49], using the Painlevé analysis, it is proved that Eq. (44) is not integrable, and an exhaustive list of analytical solutions is given. In the frame of the optics of FCPs, the field $f$ must vanish at infinity. With this condition, there is no exact analytical solution to Eq. (44) but the solutions of the real mKdV equation. Indeed, setting $f=u e^{i \varphi}$, with $u=u(Z, T)$ and $\varphi$ a constant, reduces the complex mKdV Eq. (44) to the real one. All linearly polarized FCP solitons are retrieved in this way.

Their stability to a random perturbation of the polarization can be tested numerically. If we add to the constant $\varphi$ a random noise (we used an amplitude of $0.1 \times 2 \pi$ ), it is obtained that the pulse is not destroyed, and that its polarization remains linear. However, the direction of the linear polarization slowly rotates around the propagation direction.

More interesting would be a CP soliton, of the form

$f=u(T-w Z) e^{i(\omega T-k Z)}$.

However no exact, even numerical, steady state solution of this type do exist. To be ensured of this, just plug $f$ given by the expression (46) in Eq. (44); separating real and imaginary parts and integrating once yields

$$
\begin{aligned}
& \left(3 \omega^{2}-w\right) u=u_{T T}+u^{3} \\
& \left(\omega^{2}-\frac{k}{\omega}\right) u=3 u_{T T}+u^{3}
\end{aligned}
$$

which are not compatible. However, solutions having approximately the form (46) exist and are very robust. They are studied in detail in the next section.

\section{Circularly polarized few-optical-cycle solitons}

\subsection{Analytic approximate solution valid for long pulses}

We will compute an approximate solution to the cmKdV Eq. (44), valid for large pulses, i.e. in the SVEA. Next we introduce again a small parameter $\epsilon$ and the slow variables

$\xi=\epsilon^{2} Z, \quad \eta=\epsilon(T-w Z)$,

and expand $f$ as

$f=\epsilon\left(f_{0}(\eta, \xi)+\epsilon f_{1}(\eta, \xi)+\ldots\right) e^{i(\omega T-k Z)}$,

and run the perturbative reduction procedure [42]. 
At leading order $\epsilon$, we get $k=\omega^{3}$; at second order, we find the inverse velocity $w=3 \omega^{2}$, and at order $\epsilon^{3}$ we get a nonlinear Schrödinger (NLS) equation for $f_{0}$ :

$i \partial_{\xi} f_{0}+3 \omega \partial_{\eta}^{2} f_{0}+\omega f_{0}\left|f_{0}\right|^{2}=0$

Let us consider the fundamental soliton solution of NLS:

$f_{0}=p \sqrt{6} \operatorname{sech}(p \eta) e^{i 3 p^{2} \omega \xi}$

Coming back to the initial variables, we obtain

$f=b \sqrt{6} \operatorname{sech}\left[b\left(T-3 \omega^{2} Z\right)\right] e^{i \omega\left[T-\left(\omega^{2}-3 b^{2}\right) Z\right]}$.

Eq. (52) gives an approximate solution to the cmKdV Eq. (44), which is valid for long pulses $(b \ll \omega)$.

The numerical resolution of the $\mathrm{cmKdV}$ equation is performed using the exponential time differencing second order Runge-Kutta (ETD-RK2) method [51]. The numerical scheme does not conserve exactly the $\mathcal{L}_{2}$-norm (or energy $W$ ) of the solution, however the error remains small (typically $\Delta W / W \sim 10^{-4}$ for $Z=10,000$ ).

Due to the scale invariance of the cmKdV equation, only the ratio $b / \omega$ may modify the stability properties of the solution. Practically, we fix $b=1$ and decrease the frequency $\omega$.

Fig. 1 shows the evolution of a FCP of this form, with $b=1$ and $\omega=2$. The propagation of the linearly dispersive FCP is also shown for the sake of comparison. The FCP propagates without change in width and maximum amplitude after propagation over at least $Z=10,000$ units, however, its shape is somehow distorted after propagation. The propagation speed is also quite different from the result of the above analytical approximate solution. We computed the speed of the pulse maximum. The averaged speed is about -6.8 instead of +12 . It undergoes large fast oscillations (variance about $\sigma_{f} \simeq 1.66$ ), due to a periodical move of the maximum inside the pulse. In addition, long-range fluctuations are seen (variance $\sigma_{l} \simeq 0.18$ ), which seem to be fully random. In fact, since no steady state with linear phase exists, the pulse is not a true steady state, and consequently its velocity varies in a quite erratic way; nevertheless, it is a very robust FCP.

\subsection{A robust circularly polarized FCP soliton}

Notice that the approximate solution (52) does not have a zero mean value, except at the SVEA limit $b \ll \omega$. However the mean value of the field is conserved. It is likely that the CP FCP soliton

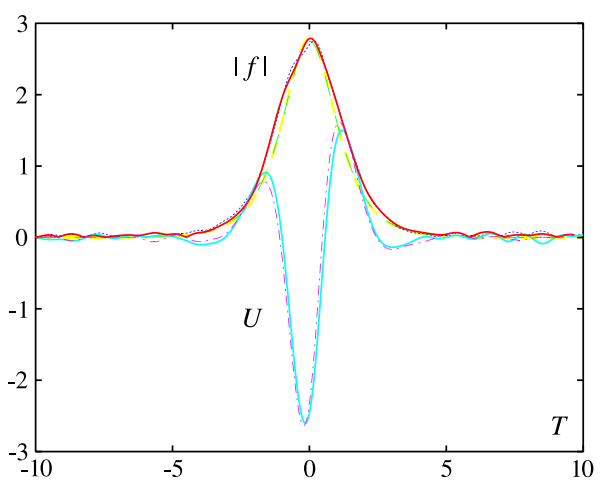

Fig. 2. Initial (at $Z=100$ ) and final (at $Z=40,000$ ) profiles of a CP FCP soliton. The initial data is composed of one mKdV breather (53) for each polarization component, with a $\pi / 2$ dephasing between them. The parameter used is $p_{1}=1+1.5 i$, which correspond to a ratio of the angular frequency divided by the pulse length of 1.5 . Blue (dotted): initial $|f|$, light blue (thick solid, gray): initial $U$, red (solid black): final $|f|$, pink (dash-dotted): final $U$. The green (thin dashed) and yellow (thick dashed, gray) lines are fits using the analytical approximate profile (52).

would have a zero mean value, and hence this would explain the discrepancy between the approximate analytical solution (52) and the direct numerical computation shown on Fig. 1.

In order to check this interpretation, let us consider an input having zero mean value. Such an alternative expression is found from the breather (or two-soliton) solution of the real mKdV equation [52]. Recall that the two-soliton has the expression

$U=\frac{e^{\eta_{1}}+e^{\eta_{2}}+\left(\frac{p_{1}-p_{2}}{p_{1}+p_{2}}\right)^{2}\left(\frac{e^{\eta_{1}}}{4 p_{1}^{2}}+\frac{e^{\eta_{2}}}{4 p_{2}^{2}}\right) e^{\eta_{1}+\eta_{2}}}{1+\frac{e^{2 \eta_{1}}}{4 p_{1}^{2}}+\frac{2 e^{\eta_{1}+\eta_{2}}}{\left(p_{1}+p_{2}\right)^{2}}+\frac{e^{2 \eta_{2}}}{4 p_{2}^{2}}+\left(\frac{p_{1}-p_{2}}{p_{1}+p_{2}}\right)^{4} \frac{e^{2 \eta_{1}+2 \eta_{2}}}{16 p_{1}^{2} p_{2}^{2}}}$,

with

$\eta_{j}=p_{j} \tau-p_{j}^{3} \zeta-\gamma_{j}$

for $j=1,2$, and becomes a breather if $p_{2}=p_{1}^{*}$. Then $\operatorname{Re}\left(p_{1}\right)$ is the inverse of the pulse length, and $\operatorname{Im}\left(p_{1}\right)$ is the angular frequency, as are $b$ and $\omega$ respectively in Eq. (52). The real part of the constant $\gamma_{1}=\gamma_{2}^{*}$ determines the position of the center of the pulse, while its imaginary part is a phase. Taking for one polarization component, say $U$, the breather (53) with $\gamma_{1 U}=0$, and for the second polarization a) Nonlinear

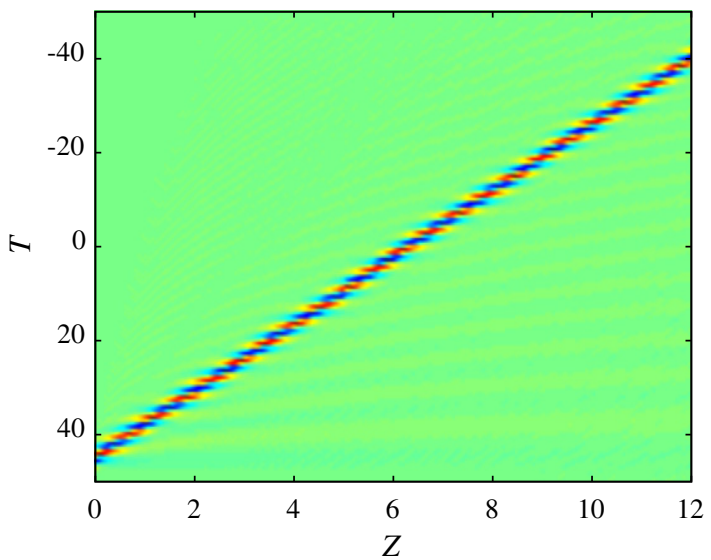

b) Linear

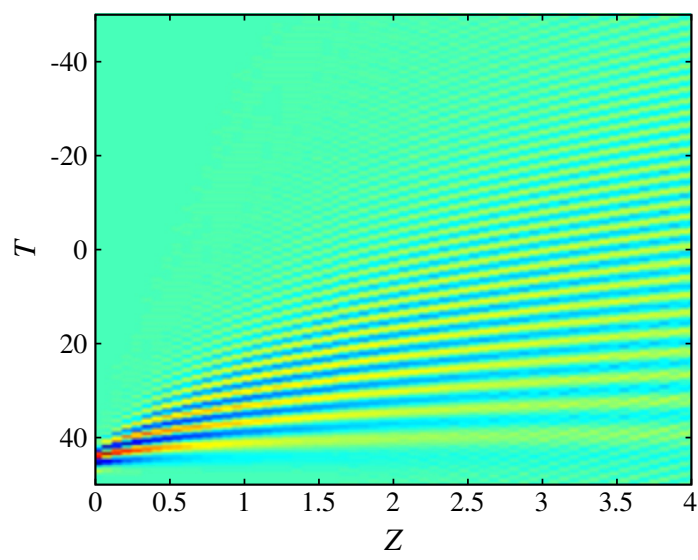

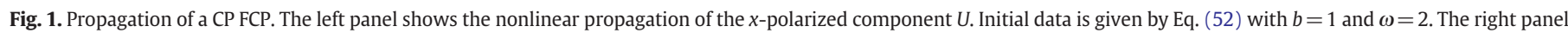
shows the propagation of the linearly dispersive FCP having the same initial profile but with very small amplitude. 


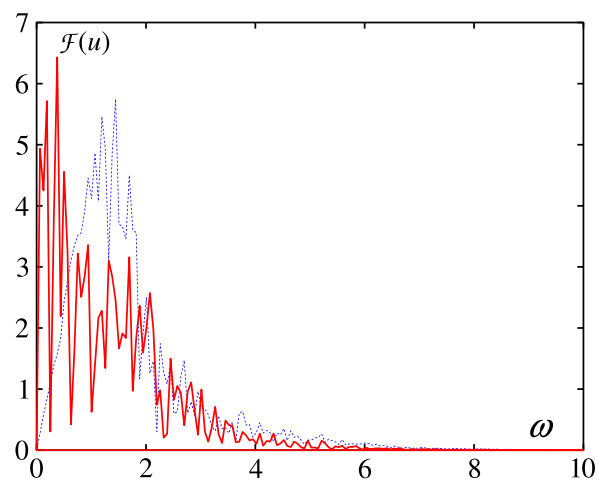

Fig. 3. Optical spectrum of an unstable CP FCP just before (at $Z=400$, blue dotted line) and just after its decay (at $Z=500$, red solid line) into slowly rotating LP half-cycle pulse. Initial data is based on Eq. (53) with $p_{1}=1+i$, which correspond to an angular frequency equal to the pulse length.

component $V$ the same expression, but with a $\pi / 2$ dephasing, i.e. with $\gamma_{1 V}=i \pi / 2$, we get some expression which can be used as an input data for solving numerically the cmKdV equation. This pulse is very close to the approximate analytical solution (52), but has a zero mean value. Numerical resolution shows that the pulse, apart from small apparently chaotic oscillations, keeps its shape and characteristics during the propagation.

Fig. 2 shows the evolution of a FCP built of two breathers dephased by $\pi / 2$, for a breather parameter $p_{1}=1+1.5 i$, which corresponds roughly to $b=1$ and $\omega=1.5$. The initial amplitude $|f|$ and the real part $U$ are depicted by the blue and light blue lines, the amplitude and the real part after propagation over $Z=40,000$ are depicted in red and pink, respectively. The locations and phase have been re-set artificially to the initial value so that comparison can be made easily. The robustness of the FCP is obvious. The sech-type profiles corresponding to formula (52) in which $b$ is evaluated using the maximum value of $|f|$ obtained numerically ( $b=1.139$ instead of 1 as expected from the input) are plotted in green and yellow respectively. Although the actual FCP is not accurately sech-shaped, the agreement is quite good. Recall that the solution (52) is valid for very large ratios $\omega / b$, while in Fig. $2, \omega / b \simeq 1.5$.

\subsection{Transition to a half-cycle soliton}

The value $\omega / b \simeq 1.5$ appears to be the lower limit for the stability of the CP FCP soliton. For smaller values of the ratio $\omega / b$, the FCP becomes unstable, and decays into a LP single-humped (half-cycle) pulse, in the form of a fundamental soliton of the real mKdV equation.

a) Initial (at $Z=100)$

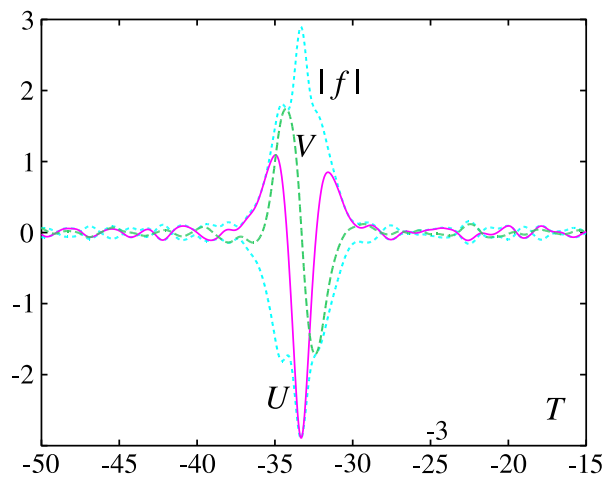

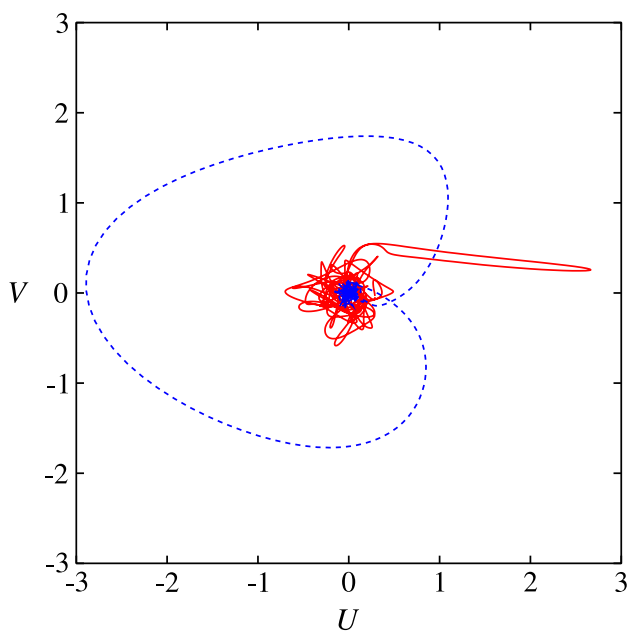

Fig. 5. Evolution of the polarization of the unstable CP FCP with both angular frequency and pulse length 1 . Blue (dotted): initial $C P$ pulse (at $Z=100$ ), red (solid): final linearly polarized pulse (at $Z=1300$ ).

The transition occurs, for $\omega / b=1.4$, between $Z=19,100$ and 19,200 , for $\omega / b=1.3$, between $Z=9300$ and 9400 , but for $\omega / b=1$, between $Z=400$ and 500 . Further, it occurs very abruptly, and involves a strong modification of the spectrum. Fig. 3 gives the evolution of the optical spectrum, i.e. the Fourier transform $\mathcal{F}(U)$ of the $x$ polarization component, in the latter case $(\omega / b=1)$. The initial spectrum is centered about $\omega / b=1$, while the second one is centered at zero.

The transition to a half-cycle soliton is shown of Figs. 4 and 5. It is a single pulse, whose profile accurately coincides with that of the fundamental soliton solution to the real $\mathrm{mKdV}$ equation

$U=\sqrt{2} b \operatorname{sech}\left(b T-b^{3} Z\right)$,

but which slowly rotates around the propagation axis. Fig. 6 show the oscillations of the two polarization components $U$ and $V$, and the evolution of the phase. The period of oscillation is close to the length of the propagation distance interval presented in the figure, about 50, which yields an 'angular frequency' $\omega \simeq 2 \pi / 50 \simeq 0.13$. In some sense, $\omega$ has fallen about one order of magnitude. However, this should not be considered as a frequency change, since $\omega$ does not represent any more the central frequency of the pulse. This rotation speed is not modified during the propagation from $Z=2000$ to $Z=10,000$ at least. However, it is strongly depending

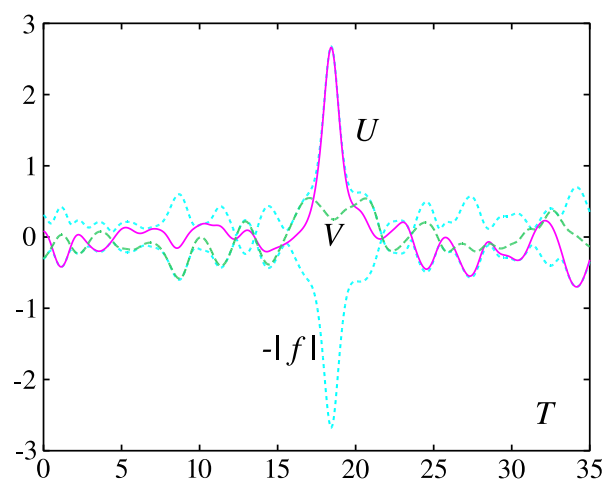

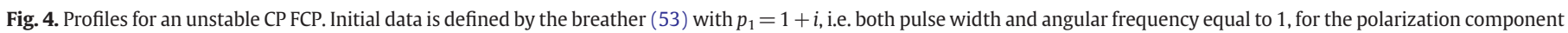
$U$, and the same with a $\pi / 2$ dephasing for $V$. Light blue (dotted): $|f|$ and $-|f|$, pink (solid): $U=\operatorname{Re}(f)$, green (dashed): $V=\operatorname{Im}(f)$. 
a) 'Initial' state, $\mathrm{Z}=2000$

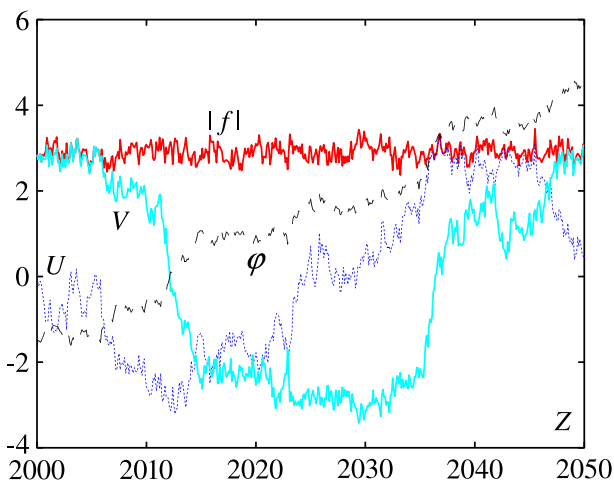

b) 'Final' state, $\mathrm{Z}=20000$

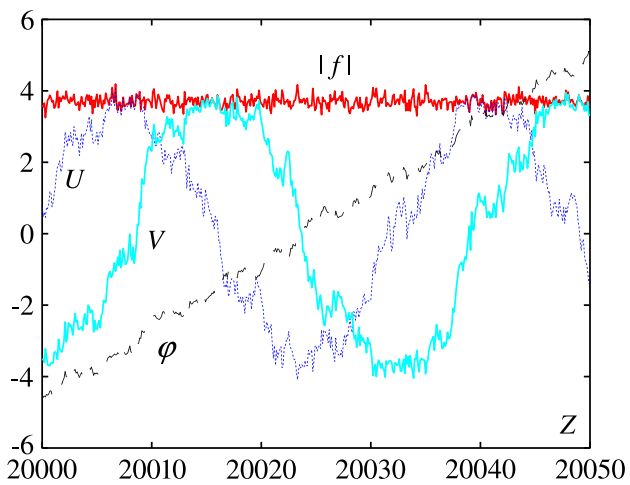

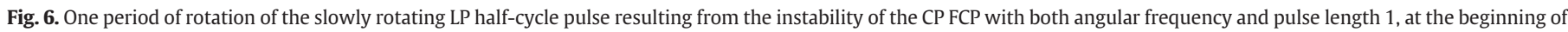

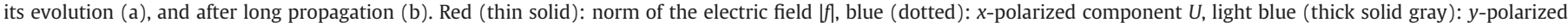
component $V$, black (dashed): phase $\arctan (V / U)$.

on the continuous pedestal. Indeed, if we remove this pedestal by setting the field $f$ to zero out of the main pulse and then compute the evolution, the rotation does not appear any more. Starting from an initial data in the form of a fundamental soliton of the real mKdV multiplied by some small linear phase,

$f(T, 0)=\sqrt{2} b \operatorname{sech}(b T) e^{i \omega T}$,

(we used $b=1$ and $\omega=0.008$ ), we get that the rotation speed gradually decreases down to zero, and the pulse becomes a fundamental soliton of the real $\mathrm{mKdV}$ equation.

We note that half-cycle optical solitons were also put forward in quadratic nonlinear media; thus a few-cycle pulse launched in a quadratic medium may result in a half-cycle soliton in the form of a single hump, with no oscillating tails [53]. We also mention here the incentive earlier works by Kazantseva et al. [54-56] on propagation and interaction of extremely short electromagnetic pulses in quadratic nonlinear media. In Ref. [56] the problem of propagation of extremely short unipolar electromagnetic pulses (the so-called "videopulses") was considered in the framework of a model in which the material medium is represented by anharmonic oscillators (approximating bound electrons) with both quadratic and cubic nonlinearities. Two families of exact analytical solutions (with positive or negative polarity) were found for the moving solitary pulses. Those videopulses were very robust against perturbations. Moreover, it was found in Ref. [56] that two such unipolar pulses collide nearly elastically, while collisions between pulses with opposite polarities and a small relative velocity are inelastic, leading to emission of radiation and generation of a small-amplitude additional pulse.

\section{Conclusion}

The multiscale perturbation analysis was used to derive approximate evolution equations governing the propagation of circularly polarized femtosecond optical solitons in cubic (Kerr-like) media beyond the slowly varying envelope approximation. Thus we took into account the vectorial character of the electric field and therefore we properly considered the wave polarization effects. In the longwave-approximation regime we have found that the two interacting wave forms corresponding to such vector few-optical-cycle solitons are adequately described by a coupled pair of complex modified Korteweg-de Vries equations at the third-order approximation of the perturbation approach. The present study completes a recent previous brief work [39] and presents an alternative derivation of the governing model in the relevant case of a "crystal-like" structure as opposed to the "glass-like" model, which was introduced and investigated in detail in Ref. [39].
A challenging extension suggested by the present work is to consider the case of two transitions, one below and one above the range of propagated wavelengths. Another interesting issue is the generalization of the present work to one or even to two spatial transverse dimensions, in addition to time and spatial longitudinal coordinates, that is, the study of formation and robustness of vector few-optical-cycle spatiotemporal solitons, alias ultrashort vector light bullets, beyond the slowly varying envelope approximation (for overviews of recent studies of spatiotemporal solitons in several relevant physical settings, see Refs. [57,58]).

\section{Acknowledgements}

The work of DM was supported in part by a Senior Chair Grant from the Région Pays de Loire, France. Support from Romanian Ministry of Education and Research (Project PN-II-ID-PCE-2011-3-0083) is also acknowledged.

\section{References}

[1] L. Gallmann, D.H. Sutter, N. Matuschek, G. Steinmeyer, U. Keller, C. Iaconis, I.A Walmsley, Optics Letters 24 (1999) 1314

[2] U. Morgner, F.X. Kärtner, S.H. Cho, Y. Chen, H.A. Haus, J.G. Fujimoto, E.P. Ippen, V. Scheuer, G. Angelow, T. Tschudi, Optics Letters 24 (1999) 411.

[3] D.H. Sutter, G. Steinmeyer, L. Gallmann, N. Matuschek, F. Morier-Genoud, U. Keller, V. Scheuer, G. Angelow, T. Tschudi, Optics Letters 24 (1999) 631.

[4] A. Shirakawa, I. Sakane, M. Takasaka, T. Kobayashi, Applied Physics Letters 74 (1999) 2268

[5] M. Wegener, Extreme Nonlinear Optics, Springer, Berlin, 2005.

[6] E. Goulielmakis, M. Schultze, M. Hofstetter, V.S. Yakovlev, J. Gagnon, M. Uiberacker A.L. Aquila, E.M. Gullikson, D.T. Attwood, R. Kienberger, F. Krausz, U. Kleineberg, Science 320 (2008) 1614.

[7] A. Scrinzi, M.Yu. Ivanov, R. Kienberger, D.M. Villeneuve, Journal of Physics B: Atomic, Molecular and Optical Physics 39 (2006) R1.

[8] T. Brabek, F. Krausz, Reviews of Modern Physics 72 (2000) 545.

[9] X. Tan, X. Fan, Y. Yang, D. Tong, Journal of Modern Optics 55 (2008) 2439

[10] N.N. Rosanov, V.E. Semenov, N.V. Vyssotina, Laser Physics 17 (2007) 1311 (ibid). Few-cycle dissipative solitons in active nonlinear optical fibres, Quantum Electron. 38 (2008) 137.

[11] A. Nazarkin, Physical Review Letters 97 (2006) 163904

[12] A.I. Maimistov, Quantum Electronics 40 (2010) 756.

[13] T. Brabec, F. Krausz, Physical Review Letters 78 (1997) 3282.

[14] M.V. Tognetti, H.M. Crespo, Journal of the Optical Society of America B: Optical Physics 24 (2007) 1410.

[15] A.A. Voronin, A.M. Zheltikov, Physical Review A 78 (2008) 063834.

[16] A. Kumar, V. Mishra, Physical Review A 79 (2009) 063807.

[17] E.M. Belenov, A.V. Nazarkin, JETP Letters 51 (1990) 288; A.I. Maimistov, S.O. Elytin, Journal of Modern Optics 39 (1992) 2201; A.E. Kaplan, P.L. Shkolnikov, Physical Review Letters 75 (1995) 2316; S.V. Sazonov, JETP Letters 92 (2001) 361.

[18] A.I. Maimistov, Optics and Spectroscopy 76 (1994) 569; Optika i Spektroskopiya 76 (1994) 636.

[19] I.V. Mel'nikov, D. Mihalache, F. Moldoveanu, N.-C. Panoiu, Physical Review A 56 (1997) 1569 (ibid);Non-envelope formulation for femtosecond optical pulses in semiconductors, JETP Lett. 65 (1997) 393.

I.V. Mel'nikov, D. Mihalache, N.-C. Panoiu, Optics Communications 181 (2000) 345. 
[20] H. Leblond, F. Sanchez, Physical Review A 67 (2003) 013804.

[21] I.V. Mel'nikov, H. Leblond, F. Sanchez, D. Mihalache, IEEE Journal of Selected Topics in Quantum Electronics 10 (2004) 870.

[22] H. Leblond, S.V. Sazonov, I.V. Mel'nikov, D. Mihalache, F. Sanchez, Physical Review A 74 (2006) 063815.

[23] H. Leblond, I.V. Mel'nikov, D. Mihalache, Physical Review A 78 (2008) 043802; H. Leblond, D. Mihalache, Physical Review A 79 (2009) 063835; H. Leblond, D. Mihalache, Journal of Optoelectronics and Advanced Materials 12 (2010) 1.

[24] S.A. Skobelev, D.V. Kartashov, A.V. Kim, Physical Review Letters 99 (2007) 203902.

[25] Sh. Amiranashvili, A.G. Vladimirov, U. Bandelow, Physical Review A 77 (2008) 063821

[26] L. Bergé, S. Skupin, Physical Review Letters 100 (2008) 113902.

[27] H. Leblond, D. Kremer, D. Mihalache, Physical Review A 80 (2009) 053812.

[28] H. Leblond, D. Kremer, D. Mihalache, Physical Review A 81 (2010) 033824; H. Leblond, D. Mihalache, Physical Review A 81 (2010) 063815.

[29] Xie Xiao-Tao, M.A. Macovei, Physical Review Letters 104 (2010) 073902.

[30] P. Dombi, S.E. Irvine, P. Rácz, M. Lenner, N. Kroó, G. Farkas, A. Mitrofanov, A. Baltuška, T. Fuji, F. Krausz, A.Y. Elezzabi, Optics Express 18 (2010) 24206

[31] N.N. Rosanov, V.V. Kozlov, S. Wabnitz, Physical Review A 81 (2010) 043815.

[32] H. Leblond, D. Mihalache, Journal of Physics A: Mathematical and Theoretical 43 (2010) 375205.

[33] A. Pusch, J.M. Hamm, O. Hess, Physical Review A 84 (2011) 023827.

[34] E.D. Farnum, J. Nathan Kutz, Optics Letters 35 (2010) 3033.

[35] A.V. Kim, S.A. Skobolev, Physical Review A 83 (2011) 063832.

[36] R.K. Dodd, J.C. Eilbeck, J.D. Gibbon, H.C. Morris, Solitons and Nonlinear Wave Equations, Academic Press, London, 1982.

[37] M.J. Ablowitz, H. Segur, Solitons and the Inverse Scattering Transform, SIAM, Philadelphia, 1981.

[38] K. Konno, W. Kameyama, H. Sanuki, Journal of the Physical Society of Japan 37 (1974) 171.

[39] H. Leblond, H. Triki, F. Sanchez, D. Mihalache, Physical Review A 83 (2011) 063802.

[40] H. Leblond, H. Triki, D. Mihalache, Physical Review A 84 (2011) 023833.

[41] T. Taniuti, C.-C. Wei, Journal of the Physical Society of Japan 24 (1968) 941.
[42] H. Leblond, Journal of Physics B: Atomic, Molecular and Optical Physics 41 (2008) 043001.

[43] G.M. Muslu, H.A. Erbay, Computers \& Mathematics with Applications 45 (2003) 503.

[44] H. Triki, M.S. Ismail, Applied Mathematics and Computation 217 (2010) 1540.

[45] M.V. Foursov, Journal of Mathematics and Physics 41 (2000) 6173.

[46] A. Biswas, E. Zerrad, A. Ranasinghe, Applied Mathematics and Computation 217 (2010) 1491.

[47] Ö. Akin, E. Özuğurlu, Mathematical Problems in Engineering 2009 (2009) 935030.

[48] B.A. Malomed, J. Fujioka, A. Espinosa-Ceron, R.F. Rodriguez, S. González, Chaos 16 (2006) 013112

[49] A.A. Mohammad, M. Can, Journal of Physics A: Mathematical and General 28 (1995) 3223.

[50] H. Zhang, Communications in Nonlinear Science and Numerical Simulation 13 (2008) 1776.

[51] S.M. Cox, P.C. Matthews, Journal of Computational Physics 176 (2002) 430.

[52] R. Hirota, Bäcklund transformations, the inverse scattering method, solitons, and their applications (Workshop Contact Transformation, Vanderbilt Univ., Nashville, Tenn., 1974, Lecture Notes in Math, 515, 1976, p. 40.

[53] H. Leblond, Physical Review A 78 (2008) 013807.

[54] E.V. Kazantseva, A.I. Maimistov, Physics Letters A 263 (1999) 434.

[55] E.V. Kazantseva, A.I. Maimistov, Quantum Electronics 30 (2000) 623.

[56] E.V. Kazantseva, A.I. Maimistov, B.A. Malomed, Optics Communications 188 (2001) 195.

[57] B.A. Malomed, D. Mihalache, F. Wise, L. Torner, Journal of Optics B: Quantum and Semiclassical Optics 7 (2005) R53;

D. Mihalache, Journal of Optoelectronics and Advanced Materials 12 (2010) 12.

[58] D. Mihalache, Proceedings of the Romanian Academy - Series A: Mathematics, Physics, Technical Sciences, Information Science 11 (2010) 142;

D. Mihalache, Romanian Reports in Physics 62 (2010) 99;

D. Mihalache, Romanian Reports in Physics 62 (2010) 697;

D. Mihalache, Romanian Reports in Physics 63 (2011) 9. 